\title{
Distribution Of Different SoIl Algal TaXa In Relation To Physico-Chemical Characteristics Of SoIl AT GHARBIA Governorate
}

\author{
M. E. H. Osman, A. El-Naggar, H.H. Omar and G.H. Esmail \\ Botany Department, Faculty of Science Tanta University Tanta, Egypt.
}

\begin{abstract}
The algal flora of some cultivated soils in addition to edaphic and physicochemical characters of sols were extensively studied at four different locations in Gharbia Governorate during the period from April 1995 to March 1996. From the total count recorded (218 species) it was found that 143 species belong to cyanophyta, 60 species to chlorophyta, 13 species to Bacillariophyta and 2 species to Euglenophyta. The cyanophyta was frequently encountered constituting $65.5 \%$ of the total algal poulations of all soil samples, with predominance of filamentous oscillatoroid forms (Oscillatoria, Phormidium, Lyngbya and Nostoc). Unicellular and colonial coccoid forms mostly represented chlorophyta, constituting $27.5 \%$ of the total recorded algal population. The results also revealed that soil and algal variables varied markedly between locations. Thus, the most soil variables, soil moiture, organic matter, total organic nitrogen, carbonates, silt, clay, $\mathrm{Na}^{+}, \mathrm{Ca}^{+2}$ and $\mathrm{Cl}^{-}$ions) were correlated with cyanobacterial growth in the studied locations. Whereas, the occurance of Cholrophyta species was correlated with the most previously mentioned variables in addition to phosphorous. AT the same time, the growth of Bacillariophyta species was correlated with moisture content, $\mathrm{pH}$ value, carbonates, sulphates and phosphorous. While, euglenophytes were dependent on organic matter, silt, clay, total $\mathrm{N}, \mathrm{Na}^{+}$and $\mathrm{K}^{+}$ions in their maintenance.
\end{abstract}

\section{Introduction}

Egyptain soil algal flora had been studied by El-Ayouty and Ayaad (1972) and Kobbia and El-Batanouny (1975) and they described some species related to Cyanophyta, Chlorophyta and Bacillariophyta .In addition, El-Kheir and Mekkey (1987) identified 49 Egyptian taxa belonging to Bacillariophyta, Cyanophyta, Chlorophyta and Xanthophyta. Recently, Ahmed (1994) represented the first attempt to survey the algal populations inhabiting some cultivated soils in upper Egypt.

The abundance of soil algae especially cyanobacteria on both cultivated and uncultivated soil is closely related to the amount of nessary mineral salts and water content of soil (Ohtani et al., 1991). With favorable $\mathrm{pH}$ and moisture conditions, the nutrient content tends to determine the luxuriance of the algal flora and the number of species present (Hong et al., 1992). Cyanobacteria are primarily photoautotrophic microorganisms, and cores through the soil show that they are usually located in the top layer of the soil and the density falls off very rapidly with depth (Fogg et al., 1973) Rodrignez et al. (1990) recorded that 
cyanobacteria require $\mathrm{Ca}^{+2}$ as a macro-nutrient for growth and nitrogen fixation, but require $\mathrm{Na}^{+}, \mathrm{CO}^{+2}$ and molybdenum $\mathrm{Mo}^{+2}$ as a micronutrients.

Lukesova and Hoffman (1996) reported that green algal predominate on and in acid soils due, in part, to the inhibitory of other groups of algae to live at lower nutrient levels. However, diatoms in certain soils grow only where appreciable amounts of phosphate and nitrate are available (Ghosh, 1986). Furthermore, Hoffmann (1989) showed that cyanobacteria especially filamentous forms and diatoms are common in neutral to alkaline soils

Terresterial algae are resistant to low as well as to high temperature, and live in cold and hot desert soils (Hoffmann, 1989). Furthermore, the soil texture interferes in the selection and distribution of soil algae. The presence of fine particles in one type of soil more than the other leads to the availability of more total exposed surface in such soil over those possessing coarse soil particles (Salama et al., 1973). Moreover, the fine particles of soil retain more moisture and more inorganic and organic nutrients needed by the algal growth as well as the plant growth.

This study was carried out to study the possible monthly changes in distribution and composition of algal flora in different type of cultivated soils in Gharbia Governorate. In addition, to study to what extent the algal flora affected by the physical and chemical characters of the different soil types

\section{Materiales and methods The study areas:}

The area chosen for the present study is a part of the Nile Delta region of Egypt known as "Gharbia Governorate". It lies between 30 30' and 31 $30^{\circ}$ altitude lines. Four locations (Samannoud, Zefta, Kafr-Elzayat and Tanta) were chosen for studying the seasonal fluctuation of soil algal distribution for a period of one year from April 1995 till March 1996. In relation to the distance from the water body (Nile River) and according to the soil type, each studied location was sampled into four stands A, B, C and D. Stands A were always the nearest to the river while stands $\mathrm{D}$ were always the most far inside the studied locations.

\section{Soil sampling:}

Soil sampling was carried out monthly from the sixteen stands for a period of one year from April 1995 till March 1996. Soil samples at each stand were collected from the surface down to a depth of $5 \mathrm{~cm}$ since it has been established (Petersen, 1935; Fritsch, 1936) that at least in the agricultural plots, the algae on the soil surface are the same as those that may be found below. Samples were then brought immediately to the laboratory, spreaded over clean sheets of paper till air-dryness, and then mixed thoroughly together. They were sieved with $2 \mathrm{~mm}$ sieve in order to get rid of debrisand, and then stored in air light plastic bags, ready for chemical analysis and isolation of algae (John, 1942; Salama and Kobbia, 1982). 


\section{Cultivation and isolation of algae:}

The following techniques were applied on air-dried soil samples for isolation and cultivation of the algae:

a- One gram of each soil samples was spread in the form of strips in Petri dishes containing sterile Z-medium (Staub, 1961). Three replicates of each soil samples were prepared. The dishes were then incubated at room temperature in a culture chamber provided with continuous fluorescent light $\left(5 \times 10^{-3} \mathrm{Lux}\right)$.

b- As described by Bold (1970), sterilized petri-dishes of 1.5-2cm deep and 9$10 \mathrm{~cm}$ diameters. These were filled with the soil to about $1 \mathrm{~cm}$ high. The soil was moistened thoroughly with sterile distilled water, then covered with chemically pure filter paper and the surface was plane with sterile spatula. The Petri-dishes were incubated at room temperature in a culture chamber and the filter paper was kept always moistened. The algal colonies were grols and then picked up, conted and identified. The identified species were then transferred to fresh solid Petridishes containing Z-medium and exposed to repeated subcultures several times. Algae were identified according to Desikachary (1959); Chapman (1962); Prescott (1962, 1970); Bold and Wynne (1985); Ashley et al., (1985); Watanabe and Niiyma (1990) and Watanabe (1992).

\section{Physical analysis of soil:}

1- Soil texture: Composition was determined using the Bouyoucus hydrometer method, whereby the percentage of sand, silt and clay were estimated (Piper, 1974).

2- Soil hygroscopic moisture content it was determined by oven-drying of a known weight (10 grams) air soil samples at $105 \mathrm{C}^{\circ}$ until constant weight. The percentage of moisture content was calculated.

\section{Chemical analysis of soil:}

1- Organic matter:

The total organic matter of the air-dried soil samples was determined by loss-on-ignition at $450 \mathrm{C}^{\circ}$ (Allen et al., 1974).

\section{2- pH value and electrical conductivity (EC):}

The soil reaction $(\mathrm{pH})$ and electrical conductivity $(\mathrm{EC})$ were determined in a 1:5 soil- water extract. $\mathrm{pH}$ values were determined using WTW TYP E50 pH conductivity meter. Electrical conductivity was measured using WTW KLE I/T.

\section{3- Inorganic elements:}

The concentration of sodium $(\mathrm{Na})$, potassium $(\mathrm{K})$, calcium $(\mathrm{Ca})$, magnesium $(\mathrm{Mg})$ and phosphorus $(\mathrm{P})$ were determined in soil extracts of $2.5 \%$ V/V glacial acetic acid. Na, K, and Ca were estimated as described by Allen et al., (1974).

\section{4- Total organic nitrogen:}

Total organic nitrogen, was determined by digested solutions of air-dried soil samples were prepared using micro-kjeldahl apparatus. Indo-phenol blue method, adopted by Allen et al., 1974, was used for total $\mathrm{N}$ determination using (4049 LKB Novasped) spectrophotometer. 


\section{5- Inorganic nitrogen (as ammonium):}

Ammonium $\left(\mathrm{NH}_{4}^{+}\right)$was estimated using Indo-phenol blue method described by Allen et al., (1974).

6- Inorganic nitrogen (as nitrates):

Nitrate was determined using sodium-Salicylate method (Deutsche Einheistverfahren Zur Wasser, Abwasser und Schlammunter Suchung (1960).

7- Determination of soluble chlorides, carbonates, bicarbonates and sulphates:

These salts estimated by the method described in Allen et al., (1974, 1989).

Statastical analysis:

The data were statisticaly analysed by using t-test (SPSS statistical PC program, 1997). Person correlation coefficient (r) and one way analysis of variance (ANOVA) were carried out using SPSS statistical PC program (1997).

\section{Results}

Qualitative distribution of soil algae:

The recorded algal flora inhabiting the soil samples were cyanophyta, chlorophyta, Bacillariophyta. As shown in Table 1 a total of 218 taxa (212 species) belonging to 70 different algal genera were identified. The algal community was quite diversified with cyanophyta 143 taxa (139 species), chlorophyta 60 taxa (60 species) and bacillariophyta 13 taxa (11 species) as the main algal group. Euglenophyta were represented only be two taxa belonging to 2 species.

Table 1: Number of genera, species and taxa per each algal group recorded within the different locations in Gharbia Governorate during the period from April 1995 to March 1996.

\begin{tabular}{|l|c|c|c|}
\hline Division & No. of Genera & No. of species & No. of Taxa \\
\hline Cyanophyta & 24 & 139 & 143 \\
Chlorophyta & 39 & 60 & 60 \\
Bacillariophyta & 6 & 11 & 13 \\
Euglenophyta & 1 & 2 & 2 \\
\hline Total & 70 & 212 & 218 \\
\hline
\end{tabular}

Regarding the number of recorded algal taxa, location IV was found to be the richest one (289 taxa) followed by location III (269 taxa). Meanwhile, location II was in the third rank (267 taxa) (Table 2). However, the least number of taxa was relatively recorded within location I (229 taxa). It can be observed that, cyanophyta followed by chlorophyta formed the most dominant algal group Egyptian J. of Phycol. Vol. 4(1), 2003 
in the studied locations. The largest number of cyanophytes was recorded within stand D of location IV (cultivated with wheat followed by rice then onion), while the lowest number was found at location I stand A (table 2). Nevertheless, stands IV D and ID (cultivated with clover, rice, then clover) were rich in chlorophyta, mean while the uncultivated stand IA was the poor one. Concerning, Bacillariophyta the greatest number of taxa was observed within location IV. However, two species of euglenophytes were found only at stands II A (cultivated with plant-egg, corn then clover) and IV D (cultivated with wheat, rice then onion).

Table (2) Number of different algal taxa recorded within the different lacations (I, II, III \& IV) in Gharbia Governorate during the period from April 1995 to March 1996.

\begin{tabular}{|c|c|c|c|c|c|c|}
\hline \multirow[b]{2}{*}{ 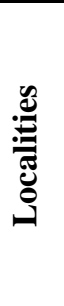 } & \multirow[b]{2}{*}{ 䔍 } & \multicolumn{4}{|c|}{ Algae } & \multirow[b]{2}{*}{$\stackrel{\bar{\Xi}}{0}$} \\
\hline & & 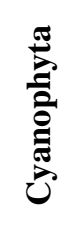 & 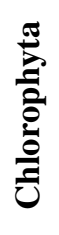 & 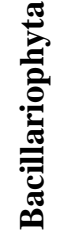 & $\begin{array}{l}\frac{\pi}{2} \\
\frac{\pi}{2} \\
\frac{0}{0} \\
\frac{0}{00} \\
\frac{0}{0}\end{array}$ & \\
\hline \multirow{5}{*}{$\mathbf{I}$} & A & 10 & 5 & 3 & -- & 18 \\
\hline & B & 42 & 14 & 4 & -- & 60 \\
\hline & $\bar{C}$ & 54 & 16 & 3 & -- & 73 \\
\hline & $\bar{D}$ & 57 & 17 & 4 & -- & 78 \\
\hline & Tot. & 163 & 52 & 14 & -- & 229 \\
\hline \multirow{5}{*}{ II } & $\overline{\mathbf{A}}$ & 44 & 12 & 4 & 2 & 62 \\
\hline & B & 54 & 14 & 3 & -- & 71 \\
\hline & $\mathbf{C}$ & 45 & 14 & 4 & 1 & 64 \\
\hline & $\bar{D}$ & 51 & 15 & 4 & -- & 70 \\
\hline & Tot. & 194 & 55 & 15 & 3 & 267 \\
\hline \multirow{5}{*}{ III } & $\overline{\mathbf{A}}$ & 59 & 16 & 2 & -- & 77 \\
\hline & $\bar{B}$ & 46 & 11 & 3 & -- & 60 \\
\hline & $\bar{C}$ & 52 & 16 & 6 & -- & 74 \\
\hline & $\bar{D}$ & 43 & 12 & 3 & -- & 58 \\
\hline & Tot. & 200 & 55 & 14 & -- & 269 \\
\hline \multirow{5}{*}{ IV } & $\overline{\mathbf{A}}$ & 48 & 15 & 7 & -- & 70 \\
\hline & $\bar{B}$ & 44 & 11 & 7 & -- & 62 \\
\hline & $\bar{C}$ & 45 & 14 & 6 & -- & 65 \\
\hline & $\overline{\mathbf{D}}$ & 65 & 18 & 7 & 2 & 92 \\
\hline & Tot. & 202 & 58 & 27 & 2 & 289 \\
\hline
\end{tabular}


Frequency of algal taxa occurrence: According to the frequency of occurrence represents the high occurrence of the following cyanophytes: Calothrix marchia, Chroococcus minutus, Cylindrospermum muscicola, Lyngbya lagerheimii, L. Limnetica, L. nordgardii, Nostoc paludosum, Oscillatoria foreauii, $O$. angustissima, $O$. subbrevis, $O$. linetica, Phormidium ambignum and Ph. tenue. On the other side, the most dominant species of chlorophyta among the different locations were: Chlorella vulgaris, Chlorococcum humicola, Coelastrum microparum, Palmella mucosa and Selenastrum minutum. Concerning Bacillariophyta, the dominant species of high occurrence were: Achnanthes linearis, Navicula mutica, Nitzschia palea, Synedra ulna and S. ulna var impressa. Mean while, rarly frequency of Euglenophyta was only recorded at two locations (II \& IV).

The monthly variation in the number of algal taxa as they appeared in the different locations. Among all of the experimental period, the greatest number of the total algal taxa was recorded in location IV, especially during the period from July 95 to January 96 . This location was followed by location III, where the maximum fluorishment was observed from August 95 to February 96 except October and November 96. However, the lowest number of total algal taxa was detected within location I from July to September 95. Mean while, the high, occurrence of algal taxa within location II was recorded during January, February and March 96. With regard to cyanophyta, location III was the richest with algal taxa, followed by location II, IV and I, respectively throughout the year (Table 3). The maximum number of algal taxa was observed within all locations, especially location IV D which was cultivated with corn during the period from June to September 95. Whereas, the lowest number of taxa was noticed in April and May 95 within location I, especially stand D (cultivated with clover).

Table 3 represents that chlorophytes were highly recorded within locations IV and III, while locations I \& II were characterized by the lowest one. Chlorophyta-fluorished mainly during the period from Decmber 95 to Febreuary 96 within all locations, especially III which was cultivated with clover.

Bacillariophyta had the greatest number of the total algal taxa within location IV, followed by location II and finally location I. However, the lowest one was recorded with in location III. The algal taxa occurred most frequently during the period from September to November 95 for all studied locations except location III which fluorished mainly in September, November 95 and March 96. In addition, April and May 95 recorded another peak for algal fluorishment in location IV and abscence for Bacillariophyta within location I.

Concerning euglenophytes, only two species were found within two locations (II \& IV) throughout the period from September to December within location II and during June and July in location IV.

Correlation between the number of algal taxa and soil variables:

The relationship between soil properties and the number of species of algal groups were correlated for different studied locations using the person 
correlation coefficient. Table 4 represented that cyanophyta species exhibited significant positive correlation with day, organic matter, total $\mathrm{N}$ and $\mathrm{Ca}^{+2}$ in locations I and IV, whereas moisture, carbonates and bicarbonates were significantly correlated with location I only. However, only the total $\mathrm{N}$ was positively correlated with location II. On the other hand, a significant negative correlation was recorded with $\mathrm{Ec}, \mathrm{NO}_{3}^{-2}, \mathrm{Na}^{+}, \mathrm{K}^{+}$and $\mathrm{Cl}^{-}$ions in location I. Chlorophyta species showed significant positive correlation with $\mathrm{pH}$, moisture and carbonates in the different locations. Also, EC, silt, organic matter and $\mathrm{Ca}^{+2}$ exhibited the same correlation in locations I and IV. Concerning Bacillariophyta species, $\mathrm{pH}$, sulphate in location I and moisture, carbonates in locations I and II showed significant positive correlations. It could be observed that $\mathrm{EC}, \mathrm{S}$ and $\mathrm{NO}_{3}{ }^{-}$ $\mathrm{Na}^{+}, \mathrm{K}^{+}$and $\mathrm{Cl}^{-}$ions were commonly of negative correlation with cyanophyta and chlorophyta. The total $\mathrm{N}$ was the only parameter which showed significant negative correlation with Bacillariophyta. For Euglenophyta species, $\mathrm{Na}^{+}$ recorded significant positive correlation, whereas a negative correlation was observed with $\mathrm{NH}_{4}$.

The analysis of variance of the soil characters and algal number of species clearly indicated that many of these variables varied significantly between locations. The overall means comparison of location I (Table 5), showed highly significant variations for $\mathrm{EC}, \mathrm{Na}^{+}, \mathrm{NO}_{3}{ }^{-}$and $\mathrm{Cl}^{-}$for stand IA, silt percentage for IB and organic matte content for ID. However, the number of algal species also of high significant relation with cyano-and chlorophyta in stand ID and Bacillariophyta in IB.

The F-value recorded for location II (Table 6) clarified that $\mathrm{pH}$, phospherous, total organic $\mathrm{N}, \mathrm{NH}_{4}, \mathrm{Cl}, \mathrm{CO}_{3}{ }^{2-}, \mathrm{HCO}_{3}{ }^{-}$and $\mathrm{SO}_{4}{ }^{2-}$ contents exhibited insignificant variation between stands. This picture affected the F-value of the number of algal species recorded in this location since it was only significant for cyamophyta species.

Regarding to values recorded for location III (Table 7), stand D was characterized by higher contents of $\mathrm{Na}^{+}, \mathrm{Ca}^{2+}$ and $\mathrm{Mg}^{2+}$ ions (salinity) than the other stands. This was supported by a high EC value in addition to higher percentage of sand, organic matter and $\mathrm{Cl}^{-}$ions.

Table 8 represents that stand $\mathrm{D}$ was the highest in silt, organic matter and $\mathrm{Ca}^{2+}$ contents. A highly significant variation was recorded for cyano-and chlorophyta number of species and most reflected also in stand D. Generally it could be observed that day, silt, sand, organic matter, $\mathrm{EC}, \mathrm{Na}^{+}, \mathrm{Cl}^{-}, \mathrm{K}^{+}, \mathrm{Mg}^{2+}$ and $\mathrm{NO}_{3}{ }^{-}$exhibited highly significant variation for all tested locations and all the $\mathrm{D}$ stands were the richest in most of the measured soil variables. 
M. E. H. Osman, et al.

Table (3) Monthly variations in the number of soil algal taxa of locations I, II, III and IV during the period from April 1995 to 1996.

\begin{tabular}{|c|c|c|c|c|c|c|c|c|c|c|c|c|c|c|c|c|c|c|c|c|c|}
\hline \multirow[t]{2}{*}{$\underset{\vec{E}}{\vec{E}}$} & \multirow[t]{2}{*}{$\vec{E}$} & \multicolumn{4}{|c|}{ 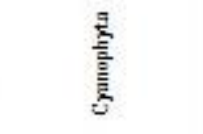 } & \multicolumn{4}{|c|}{ 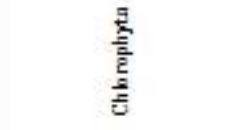 } & \multicolumn{4}{|c|}{ 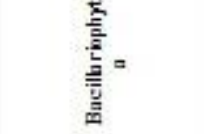 } & \multicolumn{4}{|c|}{ 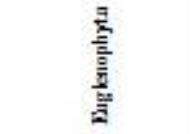 } & \multicolumn{4}{|c|}{ 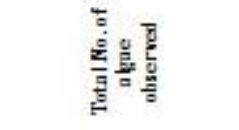 } \\
\hline & & I & II & III & IV & I & II & III & IV & I & II & III & IV & I & II & III & IV & I & II & III & IV \\
\hline & $A$ & 2 & 9 & 10 & 8 & 1 & 4 & 3 & 4 & - & - & - & 2 & - & - & - & - & 3 & 13 & 13 & 14 \\
\hline $\mathrm{g}$ & B & 10 & 9 & 8 & 9 & 2 & 2 & 2 & 3 & - & 2 & - & 3 & - & - & - & - & 12 & 13 & 10 & 15 \\
\hline 臬 & $\mathrm{c}$ & 10 & 10 & 10 & 8 & 2 & 3 & 4 & 4 & - & 2 & 1 & 3 & - & - & - & - & 12 & 15 & 15 & 15 \\
\hline & D & 11 & 10 & 7 & 12 & 4 & 3 & 4 & 4 & - & - & - & 2 & - & - & - & - & 15 & 13 & 11 & 18 \\
\hline & $\mathrm{T}$ & 33 & 38 & 35 & 37 & 9 & 12 & 13 & 15 & - & 4 & 1 & 10 & - & - & - & - & 42 & 54 & 49 & 62 \\
\hline & $A$ & 2 & 10 & 7 & 3 & 1 & 3 & 3 & 3 & $\cdot$ & - & - & 2 & - & - & - & - & 3 & 13 & 10 & 13 \\
\hline$\therefore$ & B & 10 & 10 & 6 & 10 & 2 & 2 & 2 & 3 & - & 2 & - & 3 & - & - & - & - & 12 & 14 & 8 & 16 \\
\hline$\Rightarrow$ & c & 9 & 12 & 11 & 7 & 1 & 4 & 3 & 3 & - & 1 & 1 & 3 & - & - & - & - & 10 & 17 & 17 & 13 \\
\hline 롤 & D & 3 & 11 & 10 & 10 & 3 & 3 & 4 & 3 & - & - & . & - & - & - & - & - & 11 & 14 & 14 & 13 \\
\hline & $\mathrm{T}$ & 29 & 43 & 34 & 35 & 7 & 12 & 14 & 12 & - & 3 & 1 & 8 & - & - & - & - & 36 & 58 & 49 & 35 \\
\hline & $A$ & 4 & 12 & 10 & 10 & 1 & 2 & & 4 & 1 & 2 & - & - & - & 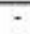 & - & - & 6 & 16 & 13 & 14 \\
\hline 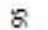 & B & 9 & 11 & 11 & 3 & 2 & 2 & 2 & 3 & 1 & - & - & - & - & - & - & - & 12 & 13 & 13 & 11 \\
\hline 站 & c & 14 & 9 & 13 & 11 & 4 & 3 & 2 & 2 & - & 1 & 1 & 2 & - & - & - & - & 18 & 13 & 16 & 15 \\
\hline & D & 17 & 12 & 13 & 15 & 4 & 3 & 2 & 5 & - & - & - & - & - & - & - & 1 & 21 & 15 & 15 & 21 \\
\hline & $T$ & 44 & 44 & 47 & 44 & 11 & 10 & 9 & 14 & 2 & 3 & 1 & 2 & - & - & $\because$ & 1 & 57 & 57 & 57 & 61 \\
\hline & $A$ & 3 & 15 & 13 & 12 & 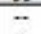 & 1 & 1 & 2 & 1 & 1 & $\because$ & - & - & - & - & - & 4 & 17 & 14 & 14 \\
\hline 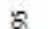 & B & 10 & 14 & 15 & 11 & 2 & 1 & 1 & 1 & 1 & 2 & - & 2 & - & - & - & $\cdot$ & 13 & 17 & 16 & 14 \\
\hline 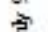 & c & 20 & 11 & 11 & 11 & 4 & 3 & 2 & 1 & : & - & 2 & - & - & - & - & - & 24 & 14 & 15 & 12 \\
\hline 主 & D & 21 & 12 & 10 & 21 & 6 & 2 & $i$ & 6 & 1 & - & $i$ & - & - & - & - & 2 & 28 & 14 & 12 & 29 \\
\hline & $\mathrm{T}$ & 54 & 52 & 49 & 55 & 12 & $?$ & 5 & 10 & 3 & 3 & 3 & 2 & - & - & - & 2 & 69 & 62 & 57 & 69 \\
\hline & $A$ & 3 & 11 & 16 & 11 & 1 & 2 & 2 & 3 & $\because$ & - & 1 & - & - & - & - & - & 4 & 13 & 19 & 14 \\
\hline 2 & B & i1 & 13 & 12 & 10 & 2 & 1 & 2 & 2 & 1 & 1 & $i$ & 2 & - & - & - & - & 14 & 15 & 16 & 14 \\
\hline th & c & 18 & 12 & 13 & 11 & 3 & 2 & 3 & 1 & - & 1 & 2 & - & - & - & - & - & 21 & 15 & 18 & 12 \\
\hline$\underline{z}$ & D & 19 & 11 & 12 & 22 & 7 & 1 & 3 & 8 & 1 & - & 1 & 3 & - & - & - & - & 27 & 12 & 16 & 33 \\
\hline & $\mathrm{T}$ & 50 & 47 & 53 & 54 & 13 & 5 & 10 & 14 & 2 & 2 & 6 & 5 & - & - & - & - & 65 & 55 & 69 & 73 \\
\hline & $A$ & 3 & 13 & 15 & 13 & 1 & 2 & 1 & 4 & 1 & 2 & 1 & 2 & - & 1 & - & - & 6 & 18 & 17 & 19 \\
\hline$g$ & B & 13 & 14 & 12 & 11 & 2 & 2 & 2 & 2 & 2 & 1 & 2 & 3 & - & - & - & - & 17 & 17 & 16 & 16 \\
\hline$=$ & $\bar{c}$ & 15 & 9 & 10 & 11 & 3 & 3 & 3 & 3 & 1 & . & 2 & 2 & - & - & - & - & 19 & 12 & is & 15 \\
\hline & D & 19 & 12 & 14 & 24 & 3 & 2 & 2 & 5 & 1 & 1 & 1 & 4 & - & - & - & - & 23 & 15 & 17 & 32 \\
\hline & $T$ & 51 & \begin{tabular}{|l|}
48 \\
\end{tabular} & 51 & 39 & 9 & 9 & 8 & 14 & 5 & 4 & 6 & 11 & - & 1 & - & - & 63 & 62 & 65 & 84 \\
\hline & $A$ & 3 & 11 & 9 & 11 & 2 & 3 & 4 & 4 & 2 & 2 & 1 & 4 & - & 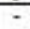 & 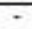 & - & 7 & 16 & 14 & 19 \\
\hline 2 & B & 11 & 11 & 10 & 9 & 3 & 3 & 3 & 3 & 3 & 1 & 2 & 2 & - & - & - & - & 17 & 15 & is & 14 \\
\hline- & c & 10 & 8 & 9 & 10 & 5 & 4 & 4 & 4 & 1 & 2 & 2 & 2 & - & 1 & - & - & 16 & 15 & 15 & 16 \\
\hline 8 & D & 12 & 9 & 9 & 16 & 3 & 3 & 5 & 3 & 1 & 1 & 2 & 2 & - & . & - & - & 16 & 13 & 16 & 21 \\
\hline & $T$ & 36 & 39 & 37 & 46 & 13 & 13 & 16 & 14 & $?$ & 6 & 3 & 10 & - & 1 & - & - & 56 & 59 & 60 & 70 \\
\hline & $A$ & 4 & 9 & 3 & 10 & 2 & 3 & 5 & 5 & $\cdot$ & 2 & - & 4 & - & 1 & - & - & 6 & 16 & 18 & 19 \\
\hline  & B & 9 & 8 & 9 & 10 & 4 & 3 & 4 & 3 & 3 & - & 1 & 2 & - & - & - & - & 16 & 15 & 14 & 15 \\
\hline & c & 10 & 9 & 10 & 3 & 4 & 4 & 4 & 4 & - & 3 & - & - & - & - & - & - & 13 & 15 & 14 & 12 \\
\hline$\frac{0}{2}$ & D & 10 & 8 & 9 & 12 & 4 & 2 & 3 & 4 & 2 & 1 & - & 3 & 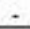 & - & - & - & 17 & 13 & 12 & 19 \\
\hline & $T$ & 33 & 35 & 41 & 40 & 14 & 12 & 16 & 16 & 3 & 5 & 1 & 9 & - & 1 & - & - & 52 & 34 & 38 & 55 \\
\hline & $A$ & 4 & 9 & 16 & 11 & 3 & 4 & 3 & 4 & - & 3 & 1 & 3 & - & - & - & - & 7 & 16 & 25 & 18 \\
\hline 8 & B & 9 & 10 & 11 & 9 & 4 & 4 & 5 & 4 & 2 & - & - & 3 & - & - & - & - & 15 & 14 & 16 & 16 \\
\hline du & $\bar{c}$ & 10 & 8 & 11 & 10 & 3 & 4 & 6 & 5 & 2 & 2 & - & 1 & - & 1 & - & - & 17 & is & 17 & 16 \\
\hline$\not{8}$ & D & 10 & 10 & 12 & 14 & 3 & 3 & 4 & 6 & 2 & 2 & - & 3 & - & - & - & - & 17 & 15 & 16 & 23 \\
\hline & $T$ & 33 & 37 & 50 & 44 & 17 & 15 & 23 & 19 & 6 & 7 & 1 & 10 & - & 1 & - & - & 56 & 60 & 74 & 73 \\
\hline & $A$ & 4 & 11 & 15 & 9 & 3 & 3 & 7 & 4 & $\because$ & 3 & 2 & 4 & - & - & 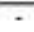 & - & 7 & 17 & 24 & 17 \\
\hline ๘ & B & 10 & 12 & 10 & 3 & 3 & 5 & 6 & 3 & 2 & 1 & - & 4 & - & - & - & $\cdot$ & 15 & 18 & 16 & 15 \\
\hline$=$ & $\mathrm{c}$ & 10 & 10 & 12 & 9 & 6 & 4 & 7 & 4 & 1 & 2 & 1 & 2 & - & - & - & - & 17 & 16 & 20 & 15 \\
\hline E & D & 9 & 12 & 9 & 11 & 4 & 3 & 4 & 3 & 3 & 3 & 1 & 3 & - & - & - & - & 16 & 20 & 14 & 19 \\
\hline & $\mathrm{T}$ & 33 & 45 & 46 & 37 & 16 & 17 & 24 & 16 & $\frac{7}{6}$ & 9 & 4 & 13 & - & - & - & - & 55 & 71 & 74 & 65 \\
\hline & $A$ & 3 & 11 & 16 & 8 & 2 & 4 & 6 & 3 & 1 & 3 & 2 & 2 & - & - & - & - & 6 & 18 & 24 & 13 \\
\hline$\approx$ & B & 10 & 10 & 9 & 7 & 3 & 3 & 4 & 4 & 3 & 1 & 2 & 1 & - & - & - & - & 16 & 14 & 15 & 12 \\
\hline 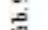 & c & 7 & 10 & 10 & 10 & 3 & 4 & 5 & 3 & 1 & 1 & 2 & 2 & - & - & - & - & 11 & 15 & 17 & 15 \\
\hline $\mathbb{E}$ & D & 9 & 10 & 3 & 14 & 4 & 4 & 3 & 3 & 1 & 3 & - & - & - & - & - & - & 14 & 17 & 11 & 19 \\
\hline & $\mathrm{T}$ & 29 & 41 & 43 & 39 & 12 & 15 & 18 & 15 & 5 & 8 & 6 & 3 & - & - & - & - & 47 & 64 & 67 & $\$ 9$ \\
\hline & & 4 & & & & 1 & 4 & 4 & 3 & 1 & 2 & $\cdot$ & 1 & 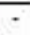 & - & - & & 6 & 20 & 18 & 10 \\
\hline & B & 9 & 13 & 11 & 6 & 3 & 4 & 4 & 3 & 2 & - & - & 1 & - & - & - & - & 14 & 17 & 15 & 10 \\
\hline$=$ & $\mathrm{c}$ & 10 & 10 & 11 & 10 & 3 & 3 & 4 & 3 & - & 2 & - & 2 & - & - & - & - & 13 & 15 & 15 & 15 \\
\hline$\frac{5}{3}$ & D & 11 & 11 & 10 & 13 & 3 & 4 & 2 & 4 & . & 2 & 1 & 1 & - & - & - & - & 16 & 17 & 13 & 18 \\
\hline & T & 34 & 43 & 46 & 35 & 10 & 15 & 14 & 13 & 5 & 6 & $i$ & 5 & - & - & - & - & 49 & 69 & 61 & 53 \\
\hline To & & $4: 4$ & $5: 5$ & 532 & 435 & 143 & $: 43$ & 170 & 170 & 47 & $6:$ & 38 & 90 & - & 4 & - & 3 & 650 & 725 & 740 & 790 \\
\hline
\end{tabular}

Egyptian J. of Phycol. Vol. 4(1), 2003 
Distribution of Different Soil Algal Taxa ...

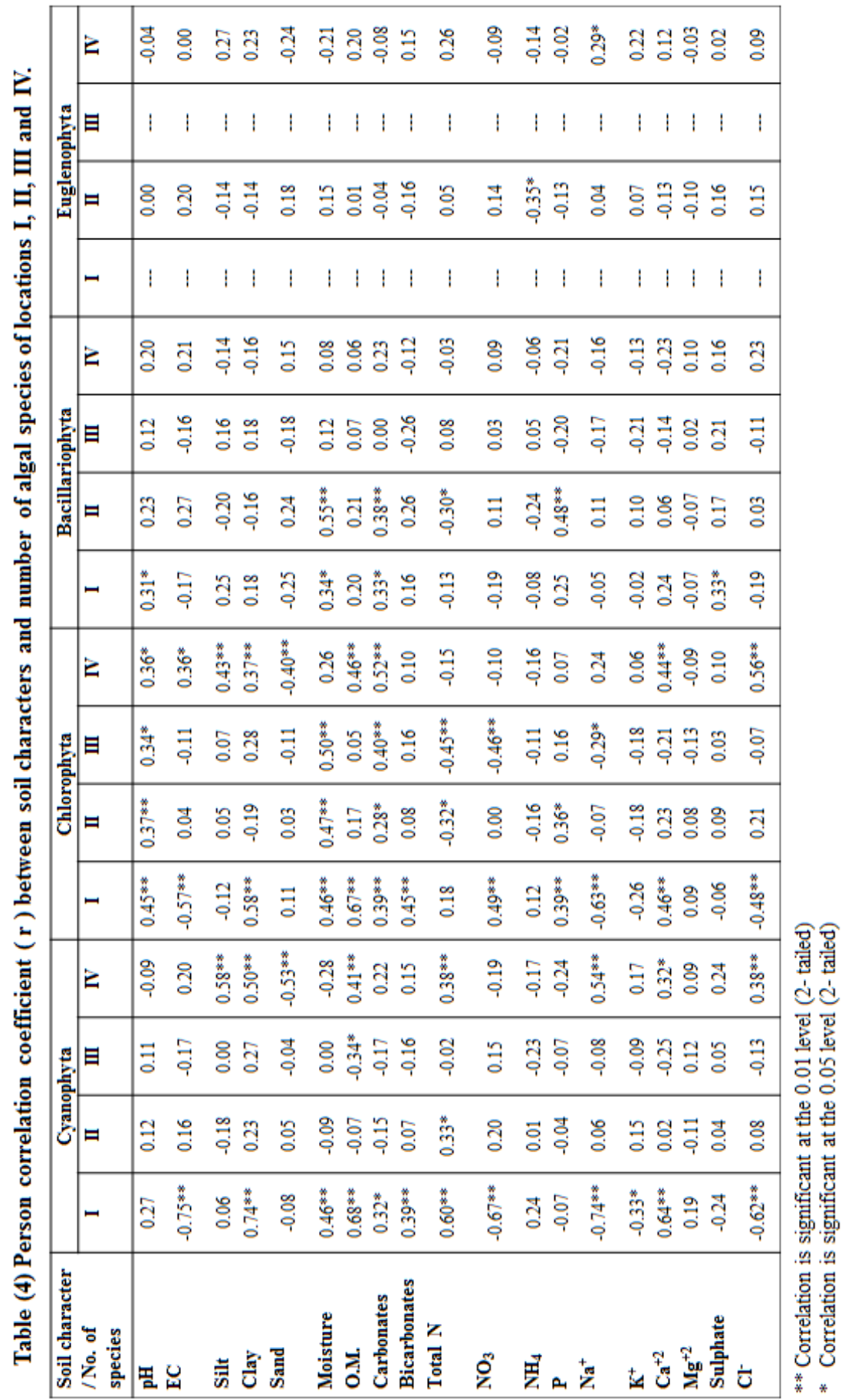


Table 5: Overall means of the physical, chemical and algal flora characteristics of soil samples at different stands of Location $\mathrm{I}^{1}$

\begin{tabular}{|c|c|c|c|c|c|c|c|}
\hline \multirow{2}{*}{\multicolumn{2}{|c|}{$\begin{array}{c}\text { Location } \\
\text { Sub - location } \\
\end{array}$}} & \multicolumn{4}{|c|}{ Location I (Samannoud) } & \multirow{3}{*}{$\begin{array}{c}\text { Mean } \pm \text { S. D } \\
20.79 \pm 0.09 \\
\end{array}$} & \multirow{3}{*}{$\begin{array}{c}\text { F-value } \\
\text { 6088.5*** } \\
\end{array}$} \\
\hline & & \multirow{2}{*}{$\frac{\mathrm{A}}{20.7}$} & \multirow{2}{*}{\begin{tabular}{c|} 
B \\
20.8 \\
\end{tabular}} & \multirow{2}{*}{$\begin{array}{c}\mathrm{C} \\
\mathbf{2 0 . 7} \\
\end{array}$} & \multirow{2}{*}{$\begin{array}{c}\text { D } \\
20.8\end{array}$} & & \\
\hline Soil & Clay & & & & & & \\
\hline Texture & Silt & 22.9 & 31.1 & 26.2 & 20.9 & $25.31 \pm 4.44$ & $1.2 \mathrm{E}+07 * * *$ \\
\hline$(\%)$ & Sand & 56.5 & 48.0 & 52.9 & 58.2 & $53.91 \pm 4.47$ & $1.3 \mathrm{E}+07 * * *$ \\
\hline \multicolumn{2}{|c|}{ Moisture Content (\%) } & 3.65 & 5.56 & 5.72 & 6.55 & $5.371 \pm 1.23$ & $12.25 * * *$ \\
\hline \multicolumn{2}{|c|}{ Organic Matter (\%) } & 5.70 & 10.12 & 11.92 & 13.52 & $10.31 \pm 3.37$ & $109.3 * * *$ \\
\hline \multicolumn{2}{|c|}{ pH value } & 7.39 & 7.88 & 7.85 & 7.93 & $7.760 \pm 0.25$ & $\mathbf{5 . 9 5} * * *$ \\
\hline \multicolumn{2}{|c|}{ EC (mmhos/cm) } & 4.13 & 0.61 & 0.36 & 0.43 & $1.380 \pm 1.83$ & $343.5 * * *$ \\
\hline \multicolumn{2}{|c|}{$\mathrm{Na}(\mathrm{mg} / \mathrm{g})$} & 8.17 & 4.42 & 2.38 & 2.83 & $4.450 \pm 2.63$ & $96.83 * * *$ \\
\hline \multicolumn{2}{|c|}{$\mathrm{Ca}$ (mg/g) } & 8.51 & 12.67 & 14.1 & 14.7 & $12.50 \pm 2.81$ & $19.76 * * *$ \\
\hline \multicolumn{2}{|l|}{$\mathrm{K}(\mathrm{mg} / \mathrm{g})$} & 1.05 & 0.86 & 0.59 & 0.69 & $0.799 \pm 0.19$ & $6.29 * * *$ \\
\hline \multicolumn{2}{|c|}{$\mathrm{Mg}(\mathrm{mg} / \mathrm{g})$} & 2.98 & 3.61 & 4.16 & 3.90 & $3.664 \pm 0.51$ & $4.98 * * *$ \\
\hline \multicolumn{2}{|l|}{$P(\mathrm{mg} / \mathrm{g})$} & 0.74 & 0.96 & 1.21 & 0.98 & $0.975 \pm 0.19$ & 1.17 \\
\hline \multicolumn{2}{|c|}{ Organic N(mg/g) } & 5.99 & 5.18 & 6.83 & 5.87 & $5.970 \pm 0.67$ & $8.13 * * *$ \\
\hline \multicolumn{2}{|c|}{$\mathrm{NH}_{4}(\mathrm{mg} / \mathrm{g})$} & 0.24 & 0.30 & 0.32 & 0.33 & $0.300 \pm 0.04$ & 2.08 \\
\hline \multicolumn{2}{|c|}{$\mathrm{NO}_{3}(\mathrm{mg} / \mathrm{g})$} & 18.2 & 3.13 & 1.24 & 0.87 & $5.868 \pm 8.30$ & 63.22*** \\
\hline \multicolumn{2}{|l|}{ CL $(\%)$} & 0.95 & 0.066 & 0.039 & 0.042 & $0.275 \pm 0.45$ & $24.1 * * *$ \\
\hline \multicolumn{2}{|l|}{$\mathrm{CO}_{3}(\%)$} & 0.002 & 0.014 & 0.013 & 0.016 & $0.011 \pm 0.01$ & $6.64 * * *$ \\
\hline \multicolumn{2}{|c|}{$\mathrm{HCO}_{3}(\%)$} & 0.08 & 0.129 & $\mathbf{0 . 1 2 7}$ & 0.137 & $0.118 \pm 0.02$ & $5.87 * * *$ \\
\hline \multicolumn{2}{|c|}{$\mathrm{S0}_{4}(\%)$} & 1.38 & 1.172 & 1.023 & 1.238 & $1.200 \pm 0.15$ & $2.82 *$ \\
\hline \multicolumn{2}{|c|}{ Cyanophyta } & 3.33 & 10.08 & 11.83 & 13.08 & $9.583 \pm 4.855$ & $23.28 * * *$ \\
\hline \multicolumn{2}{|c|}{ Chloropbyta } & 1.50 & 2.67 & 3.58 & 4.17 & $2.979 \pm 1.480$ & $13.17 * * *$ \\
\hline \multicolumn{2}{|c|}{ Bacillariophyta } & 0.53 & 1.67 & 0.50 & 1.17 & $0.979 \pm 0.956$ & 4.88*** \\
\hline
\end{tabular}

1 Values in a row followed by $*=$ significant difference at $P \leq 0.05, * *==P \leq 0.01$, $* * *=\mathbf{P} \leq \mathbf{0 . 0 0 5}$ according to $\mathbf{F}-$ test 
Table 6: Overall means of the physical, chemical and algal flora characteristics of soil samples at different stands of Location $\mathrm{II}^{1}$

\begin{tabular}{|c|c|c|c|c|c|c|c|}
\hline \multirow{2}{*}{\multicolumn{2}{|c|}{$\begin{array}{c}\text { Location } \\
\text { Sub - location }\end{array}$}} & \multicolumn{4}{|c|}{ Location II(Zefta) } & \multirow{3}{*}{$\begin{array}{c}\text { Mean } \pm \text { S. D } \\
18.45 \pm .89\end{array}$} & \multirow{3}{*}{$\begin{array}{c}\text { F-value } \\
646205 * * *\end{array}$} \\
\hline & & $\mathbf{A}$ & $\mathbf{B}$ & $\mathbf{C}$ & D & & \\
\hline \multirow{3}{*}{$\begin{array}{l}\text { Soil } \\
\text { Texture } \\
(\%)\end{array}$} & Clay & 18.3 & 19.8 & $\mathbf{1 7 . 9}$ & 17.9 & & \\
\hline & Silt & 16.8 & 20.4 & 20.7 & 19.9 & $19.46 \pm 0.79$ & $2.4 \mathrm{E}+06 * * *$ \\
\hline & Sand & 64.9 & 59.8 & 61.5 & 62.2 & $62.09 \pm .122$ & $3.6 \mathrm{E}+06^{* * * *}$ \\
\hline \multicolumn{2}{|c|}{ Moisture Content (\%) } & 6.32 & 5.88 & 6.39 & 5.85 & $6.111 \pm .283$ & 0.665 \\
\hline \multicolumn{2}{|c|}{ Organic Matter (\%) } & 10.6 & 11.6 & 9.61 & 9.35 & $10.29 \pm .039$ & $4.595 * *$ \\
\hline \multicolumn{2}{|c|}{ pH value } & 7.78 & 7.84 & 7.92 & 7.97 & $7.876 \pm .083$ & 0.648 \\
\hline \multicolumn{2}{|c|}{ EC (mmhos/cm) } & 1.38 & 0.61 & 0.49 & 0.43 & $0.727 \pm .444$ & 38.8**** \\
\hline \multicolumn{2}{|c|}{$\mathrm{Na}(\mathrm{mg} / \mathrm{g})$} & 3.66 & 2.62 & 3.92 & 3.99 & $3.544 \pm .636$ & 3.38* \\
\hline \multicolumn{2}{|c|}{$\mathrm{Ca}(\mathrm{mg} / \mathrm{g})$} & 11.2 & 12.3 & 14.8 & 11.9 & $12.57 \pm .533$ & $4.24 * *$ \\
\hline \multicolumn{2}{|l|}{$\mathrm{K}(\mathrm{mg} / \mathrm{g})$} & 1.07 & 1.00 & $\mathbf{0 . 5 7}$ & $\mathbf{0 . 5 8}$ & $0.804 \pm .269$ & $7.85 * * *$ \\
\hline \multicolumn{2}{|c|}{$\mathrm{Mg}(\mathrm{mg} / \mathrm{g})$} & 2.98 & 4.10 & 4.01 & 4.38 & $3.687 \pm .612$ & $8.96 * * * *$ \\
\hline \multicolumn{2}{|c|}{$\mathbf{P}(\mathrm{mg} / \mathrm{g})$} & 0.88 & 0.69 & 0.69 & 0.67 & $0.732 \pm .098$ & 0.847 \\
\hline \multicolumn{2}{|c|}{ Organic N(mg/g) } & 5.32 & 6.15 & 6.71 & 4.67 & $5.710 \pm .895$ & 0.929 \\
\hline \multicolumn{2}{|c|}{$\mathrm{NH}_{4}(\mathrm{mg} / \mathrm{g})$} & 0.28 & 0.28 & 0.26 & 0.29 & $0.280 \pm .015$ & 0.323 \\
\hline \multicolumn{2}{|c|}{$\mathrm{NO}_{3}(\mathrm{mg} / \mathrm{g})$} & 7.81 & 5.79 & 1.39 & 0.91 & $3.974 \pm .372$ & $7.68 * * *$ \\
\hline \multicolumn{2}{|l|}{ CL(\%) } & 0.04 & 0.04 & 0.03 & $\mathbf{0 . 0 3}$ & $0.036 \pm .006$ & 2.25 \\
\hline \multicolumn{2}{|l|}{$\mathrm{CO}_{3}(\%)$} & 0.01 & 0.01 & 0.02 & 0.01 & $0.013 \pm .001$ & 0.211 \\
\hline \multicolumn{2}{|c|}{$\mathrm{HCO}_{3}(\%)$} & 0.12 & 0.104 & 0.10 & 0.11 & $0.110 \pm .009$ & 0.702 \\
\hline \multicolumn{2}{|l|}{$\mathrm{S0}_{4}(\%)$} & 1.19 & 1.23 & 1.12 & 1.02 & $1.142 \pm .096$ & 0.943 \\
\hline \multicolumn{2}{|c|}{ Cyanophyta } & 11.25 & 11.33 & 9.83 & 10.67 & $10.771 \pm 1.692$ & 3.36* \\
\hline \multicolumn{2}{|c|}{ Chlorophyta } & 2.92 & 2.67 & 3.42 & 2.92 & $2.979 \pm 1.021$ & 1.26 \\
\hline \multicolumn{2}{|c|}{ Bacillariophyta } & 1.67 & 0.92 & 1.42 & 1.08 & $1.271 \pm 1.026$ & 1.20 \\
\hline \multicolumn{2}{|c|}{ Euglenophyta } & 0.17 & 0.00 & 0.17 & 0.00 & $0.083 \pm 0.279$ & 1.47 \\
\hline
\end{tabular}

1 Values in a row followed by $*=$ significant difference at $\mathrm{P} \leq 0.05, * *==\mathrm{P} \leq 0.01$, $* * *=\mathbf{P} \leq \mathbf{0 . 0 0 5}$ according to $\mathbf{F}-$ test 
Table 7: Overall means of the physical, chemical and algal flora characteristics of soil samples at different stands of Location III ${ }^{1}$

\begin{tabular}{|c|c|c|c|c|c|c|c|}
\hline \multirow{2}{*}{\multicolumn{2}{|c|}{$\begin{array}{c}\text { Location } \\
\text { Sub - location }\end{array}$}} & \multicolumn{4}{|c|}{ Location III ( K. Elzayat) } & \multirow{3}{*}{$\begin{array}{c}\text { Mean } \pm \text { S.D } \\
20.20 \pm 0.541\end{array}$} & \multirow{3}{*}{$\begin{array}{c}\text { F-value } \\
201918 * * *\end{array}$} \\
\hline & & $\mathbf{A}$ & B & $\mathbf{C}$ & D & & \\
\hline \multirow{3}{*}{$\begin{array}{c}\text { Soil } \\
\text { Texture } \\
(\%)\end{array}$} & Clay & 20.7 & 19.8 & 20.9 & 19.8 & & \\
\hline & Silt & 22.9 & 26.6 & 25.2 & 18.9 & $23.42 \pm 0.317$ & $9.9 \mathrm{E}+06 * * *$ \\
\hline & Sand & 56.5 & 53.6 & 53.9 & 61.2 & $56.29 \pm 3.948$ & $2.1 \mathrm{E}+06^{* * * *}$ \\
\hline \multicolumn{2}{|c|}{ Moisture Content (\%) } & 5.11 & 4.46 & 5.33 & 6.51 & $5.353 \pm 0.855$ & 3.36* \\
\hline \multicolumn{2}{|c|}{ Organic Matter (\%) } & 5.22 & 7.68 & 11.03 & 13.61 & $9.385 \pm 3.689$ & $63.8 * * *$ \\
\hline \multicolumn{2}{|c|}{ pH value } & 7.80 & 7.86 & 7.85 & 7.81 & $7.834 \pm 0.029$ & 0.123 \\
\hline \multicolumn{2}{|c|}{ EC (mmhos/cm) } & 0.32 & 0.28 & 0.32 & 2.72 & $0.910 \pm 1.205$ & $818.3 * * *$ \\
\hline \multicolumn{2}{|c|}{$\mathrm{Na}(\mathrm{mg} / \mathrm{g})$} & 2.92 & 1.77 & 2.01 & 6.46 & $3.289 \pm 2.169$ & $38.74 * * *$ \\
\hline \multicolumn{2}{|c|}{$\mathrm{Ca}(\mathrm{mg} / \mathrm{g})$} & 6.04 & 8.65 & 8.72 & 14.2 & $9.395 \pm 3.419$ & $71.45 * * *$ \\
\hline \multicolumn{2}{|c|}{$\mathrm{K}(\mathrm{mg} / \mathrm{g})$} & 0.88 & 0.85 & 0.90 & 1.12 & $0.940 \pm 0.123$ & 2.01 \\
\hline \multicolumn{2}{|c|}{$M g(\mathrm{mg} / \mathrm{g})$} & 3.94 & 3.50 & 3.55 & 4.29 & $3.820 \pm 0.368$ & $2.97 *$ \\
\hline \multicolumn{2}{|c|}{$\mathrm{P}(\mathrm{mg} / \mathrm{g})$} & 1.09 & 1.18 & 1.35 & 1.77 & $1.348 \pm 0.301$ & 2.27 \\
\hline \multicolumn{2}{|c|}{ Organic N(mg/g) } & 5.59 & 5.42 & 5.17 & 4.83 & $5.250 \pm 0.332$ & 0.222 \\
\hline \multicolumn{2}{|c|}{$\mathrm{NH}_{4}(\mathrm{mg} / \mathrm{g})$} & 0.19 & 0.21 & 0.29 & 0.29 & $0.240 \pm 0.053$ & 3.73* \\
\hline \multicolumn{2}{|c|}{$\mathrm{NO}_{3}(\mathrm{mg} / \mathrm{g})$} & 1.36 & 1.20 & 1.38 & 2.34 & $1.573 \pm 0.521$ & 1.53 \\
\hline \multicolumn{2}{|l|}{ CL(\%) } & 0.07 & 0.027 & 0.035 & 0.234 & $0.091 \pm 0.097$ & $23.05 * * *$ \\
\hline \multicolumn{2}{|l|}{$\mathrm{CO}_{3}(\%)$} & 0.01 & 0.012 & 0.01 & 0.012 & $0.010 \pm 0.002$ & 0.306 \\
\hline \multicolumn{2}{|c|}{$\mathrm{HCO}_{3}(\%)$} & 0.09 & 0.093 & 0.101 & 0.128 & $0.102 \pm 0.019$ & $7.43 * * *$ \\
\hline \multicolumn{2}{|c|}{$\mathrm{SO}_{4}(\%)$} & 1.12 & 1.039 & 1.18 & 1.52 & $1.215 \pm 0.213$ & 2.10 \\
\hline \multicolumn{2}{|c|}{ Cyanophyta } & 12.83 & 10.33 & 10.92 & 10.25 & $11.083 \pm 2.439$ & 2.16 \\
\hline \multicolumn{2}{|c|}{ Chlorophyta } & 3.92 & 3.08 & 4.08 & 3.08 & $3.542 \pm 1.663$ & 1.15 \\
\hline \multicolumn{2}{|c|}{ Bacfllariophyta } & 0.67 & 0.75 & 1.17 & 0.58 & $0.792 \pm 0.824$ & 1.31 \\
\hline
\end{tabular}

1 Values in a row followed by $*=$ significant difference at $P \leq 0.05, * *==P \leq 0.01$, $* * *=\mathbf{P} \leq \mathbf{0 . 0 0 5}$ according to $\mathbf{F}$ - test 
Table 8: Overall means of the physical, chemical and algal flora characteristics of soil samples at different stands of Location $I^{1}$

\begin{tabular}{|c|c|c|c|c|c|c|c|}
\hline \multirow{2}{*}{\multicolumn{2}{|c|}{$\begin{array}{l}\text { Location } \\
\text { Sub - location }\end{array}$}} & \multicolumn{4}{|c|}{ Location IV (Tanta) } & \multirow{3}{*}{$\begin{array}{l}\text { Mean } \pm \text { S.D } \\
19.72 \pm 2.439 \\
\end{array}$} & \multirow{3}{*}{$\begin{array}{l}\text { F-value } \\
3.9 \mathrm{E}+06 * * * \\
\end{array}$} \\
\hline & & \multirow{2}{*}{$\begin{array}{c}\text { A } \\
18.1\end{array}$} & \multirow{2}{*}{\begin{tabular}{|c|} 
B \\
17.2
\end{tabular}} & \multirow{2}{*}{$\begin{array}{c}\mathrm{C} \\
21.4\end{array}$} & \multirow{2}{*}{\begin{tabular}{|c|} 
D \\
22.2
\end{tabular}} & & \\
\hline Soil & Clay & & & & & & \\
\hline Texture & Silt & 21.6 & 21.3 & 23.6 & 24.9 & $22.86 \pm 1.739$ & $1.9 \mathrm{E}+06 * * *$ \\
\hline$(\%)$ & Sand & 60.3 & 61.5 & 54.9 & 52.9 & $57.42 \pm 4.161$ & $1.1 \mathrm{E}+06 * * * *$ \\
\hline \multicolumn{2}{|c|}{ Moisture Content (\%) } & 6.13 & 6.35 & 7.56 & 7.54 & $6.894 \pm 0.762$ & 1.45 \\
\hline \multicolumn{2}{|c|}{ Organic Matter (\%) } & 10.2 & 10.8 & 12.6 & 14.7 & $12.09 \pm 2.033$ & $30.11 * * *$ \\
\hline \multicolumn{2}{|c|}{ pH value } & 7.88 & 7.77 & 7.93 & 7.95 & $7.881 \pm .0812$ & 0.579 \\
\hline \multicolumn{2}{|c|}{ EC (mmhos/cm) } & 0.79 & 0.59 & 0.51 & 0.83 & $0.678 \pm 0.155$ & $7.03 * * *$ \\
\hline \multicolumn{2}{|c|}{$\mathrm{Na}(\mathrm{mg} / \mathrm{g})$} & 3.20 & 2.09 & 4.18 & 5.17 & $3.660 \pm 1.319$ & $21.73 * * *$ \\
\hline \multicolumn{2}{|c|}{$\mathrm{Ca}(\mathrm{mg} / \mathrm{g})$} & 13.5 & 10.3 & 10.9 & 17.9 & $13.15 \pm 3.473$ & $22.3 * * *$ \\
\hline \multicolumn{2}{|l|}{$\mathrm{K}(\mathrm{mg} / \mathrm{g})$} & 1.09 & 0.75 & 1.19 & 1.07 & $1.023 \pm 0.189$ & $5.67 * * *$ \\
\hline \multicolumn{2}{|c|}{ Mg (mg/g) } & 3.62 & 4.01 & 4.29 & 3.85 & $3.941 \pm 0.282$ & 1.95 \\
\hline \multicolumn{2}{|c|}{$P(\mathrm{mg} / \mathrm{g})$} & 0.79 & 1.14 & 1.57 & 1.31 & $1.204 \pm 0.325$ & $7.24 * * *$ \\
\hline \multicolumn{2}{|c|}{ Organic N(mg/g) } & 4.95 & 7.12 & 6.58 & 6.90 & $6.390 \pm 0.983$ & 0.927 \\
\hline \multicolumn{2}{|c|}{$\mathrm{NH}_{4}(\mathrm{mg} / \mathrm{g})$} & 0.24 & 0.33 & 0.29 & 0.26 & $0.280 \pm 0.039$ & $2.76 *$ \\
\hline \multicolumn{2}{|c|}{$\mathrm{NO}_{3}(\mathrm{mg} / \mathrm{g})$} & 5.29 & 3.24 & 1.49 & 1.05 & $2.770 \pm 1.934$ & $6.41 * * *$ \\
\hline \multicolumn{2}{|c|}{ CL(\%) } & 0.06 & 0.04 & 0.041 & 0.08 & $0.055 \pm 0.019$ & $12.81 * * *$ \\
\hline \multicolumn{2}{|l|}{$\mathrm{CO}_{3}(\%)$} & 0.01 & .008 & 0.018 & 0.02 & $0.015 \pm 0.007$ & $4.72 * *$ \\
\hline \multicolumn{2}{|c|}{$\mathrm{HCO}_{3}(\%)$} & 0.12 & 0.11 & 0.116 & 0.14 & $0.120 \pm 0.012$ & 2.63 \\
\hline \multicolumn{2}{|l|}{$\mathrm{SO}_{4}(\%)$} & 1.29 & 1.10 & 1.08 & 1.34 & $1.204 \pm 0.134$ & 0.959 \\
\hline \multicolumn{2}{|c|}{ Cyanophyta } & 9.75 & 9.00 & 9.67 & 15.33 & $10.938 \pm 3.675$ & $14.29 * * *$ \\
\hline \multicolumn{2}{|c|}{ Chiorophyta } & 3.58 & 2.83 & 3.08 & 4.83 & $3.583 \pm 1.318$ & $7.60 * * *$ \\
\hline \multicolumn{2}{|c|}{ Bacillariophyta } & 2.00 & 2.17 & 1.58 & 1.75 & $1.875 \pm 1.299$ & 0.461 \\
\hline \multicolumn{2}{|c|}{ Euglcnopbyta } & 0.00 & 0.00 & 0.00 & 0.25 & $0.063 \pm 0.320$ & 1.94 \\
\hline
\end{tabular}

1 Values in a row followed by $*=$ significant difference at $\mathrm{P} \leq 0.05, * *==\mathrm{P} \leq 0.01$,

$* * *=\mathbf{P} \leq \mathbf{0 . 0 0 5}$ according to $\mathbf{F}-$ test

\section{Discussion}

The role of edaphic factors and physico-chemical characters of the sopil is very important, not only in determining and distributing the macrovegetation and plant communities, but also the soil microorganism. The surveyed area shows an interesting and wide range of variation in both environmental conditions and algal flora distribution. Hoffmann (1989) reported that edaphic factors and soil characters together with some specific features within the algal organisms themselves constitute the main factors responsible for the existence of algae in soils. The present study showed that the majority of algal species from all sites are related to Cyanophyta, especially oscillatoroid species. Out of 218 species of algae identified, 143 species belong to Cyanophyta and 60 to Chlorophyta and 13 to Bacillariophyta and 2 species belong to Euglenophyta.

The presence of numerous Cyanophyta members as compared to other types of soil algae is a matter of tolerance and adaptability (Brock, 1973 and Metting, 1981). The organisms were found to fluorish at $\mathrm{pH}$ values of more than 7 and they are intolerant to low pH conditions. Metting, 1981 and Aziz et al., 1991). 
The $\mathrm{pH}$ values in all studied locations were more than 7 this might partially explain the wide distribution of Cyanobacteria being represented in all locations than the other algal groups. However, it was noticed from the correlation studies that though $\mathrm{pH}$ values of the all locations were positively correlated with Cyanophyta growth but this correlation was not significant. This may implicate that factors other than $\mathrm{pH}$ value might affect the distribution of Cyanophyta between locations. On the other side, the widespread of cyanophycean members in the different locations studied with relative high abundance than the other algal groups, may be due to mainly to the cellular structure of such organisms. Such assumption is in conformity with the finding of (Melting, 1981) who reported the ability of cyanobacteria to survive under variable and even adverse conidtions. The presence of higher algal populations, specially cyanobacteria in location IV may be attributed to the presence of high contents of organic matter, carbonates, $\mathrm{Ca}^{+2}, \mathrm{Na}^{+}$, and total organic $\mathrm{N}$, which reflect their significant positive effect on the growth and multiplication of the soil algal flora. These results are in agreement with those observed by Salama and Kobbia (1982).

Concerning the Chlorophyta species found in the surveyed areas, the showed significant positive correlation with moisture content, organic matter and carbonate contents in the soil as the prime factors for their growth and distribution. Chlorella vulgaris, Chloroococcum humicola and Coelastrum microporum, which appeared to be the most frequently observed and abundant green algae, have predilection for organic materials and moisture as essential factors for their growth. These observations are in conformity with the findings of Kobbia and El-Batanouny (1975) Kobbia and Shabana (1988) and Ahmed (1994). The distribution pattern of diatoms, as revealed in this investigation was of significant positive correlation with $\mathrm{pH}$ value, carbonates, sulfaes, phosphorous and moisture content, which reflect the importance of these soil parmateres for their growth. The most elaborated species were Synedra ulna impressa, Nitschia pale. Navicula mutica and Achnanthus linearis which tend to flourish in soils of relatively high $\mathrm{pH}$ values and considerably high total soluble salts. Such correlation was confirmed earlier by Metting (1981). In this connection, Dey and Ghosh (1986) reported that diatoms of certain soils grow only where appreciable amount of phosphorous and nitrates are available.

Concerning euglenoid species, they were found only in location II (stands A, C) and in location IV (stand D), which were characterized by high content of organic matter, $\mathrm{NH}_{4}{ }^{+}$content and total soluble salts especially $\mathrm{Na}^{+}, \mathrm{K}^{+}$and $\mathrm{Ca}^{2+}$ ions. These findings might indicate a response to organic and inorganbic contents of the soil and were in accordance with those observed by Pringsheim (1950), Kobbia and Shabana (1988) and Ahmed (1994).

The soil texture may also interfere in selecting and distributing soil algae. Its importance comes from the fact that the presence of fine particles $(\geq 2: 50 \mu$ in diameter) in one type of soil more than the other, leads to the availability of more total exposed surfaces in such soil over that possessing coarse soil particles (Cano 
et al., 1997). The results obtained in this study are in agreement with these findings, where location IV, especially stand D, which characterized by high silt and clay soil fractions, supported higher algal populations than the other three locations of Gharbia Governorate. Although location I was also characterized by high content of silt and clay soil fractions, it exhibited the lowest algal species numbers recorded. This may be due to the high salinity level $(\mathrm{EC}=1.4 \mathrm{mmhos} / \mathrm{cm})$ found in this location. Even in the presence of other promotive factors such as organic matter, $\mathrm{CO}_{3}{ }^{2-}$, and $\mathrm{Ca}^{2+}$ ions, which showed positive significant correlation with the algal growth in this location.

In this study it was noticed that there was a significant positive correlation between total organic nitrogen and Cyanophyta growth in location I and location IV and with available nitrogen (as $\mathrm{NO}^{3}$ ) in location II. Yjese findings may be interpreted in the light of multiple reasons. The most important one is the addition of $\mathrm{N}^{2-}$ fertilizers by the farmers during crop cultivation, especially rice have an inhibitory effect on $\mathrm{N}^{2-}$ fixing species of Cyanobacteria; Mandal et al., 1993a, b,c). Another reason is that the most dominant Cyanophycean members were the oscillatoriod forms, not the $\mathrm{N}^{2-}$ fixing species, which are not capable of nitrogen fixation. In addition, the high salinity recorded in location I that might depress the nitrogen fixation process (Rogers and Reynaud 1979).

Although there are similarities in the edaphic factors and physicochemical characters of the studied locations, I, II, III and IV of Gharbia Governorate as shown from their corresponding means, they show dissimilarities between the abundance and type of algal populations exist. This phenomenon is most probably associated with certain vegetation inhibiting the different locations (Salama and Kobbia, 1982). It should be mentioned that, there were some soil factors, such as $\mathrm{HCO}_{3}^{-}, \mathrm{SO}_{4}^{2-}, \mathrm{K}^{+}, \mathrm{Mg}^{+2}, \mathrm{NH}_{4}^{+}, \mathrm{NO}_{3}^{-}$and $\mathrm{P}$ contents, which exhibited different significant correlation among different locations for the same algal group or even among different algal groups in the same location. These factors were not found to be a prime ones affecting distribution of different algal groups and was merely contributing to those that showed significant controlling effect on algal growth between locations.

Generally, this survey revealed that members of Cyanobacteria and coccoid green algae represented the predominant forms. Such observations are in confirmation with the findings of Kobbia and Shabana, (1988) and Ahmed, (1994). Another conclusion could be mentioned that organic matter, moisture and carbonates content of soil samples seemed to be the major controlling factors affecting the growth and distribution of algae in the studied locations. These results are in consistence with those reported by Kobbia and Shabana, (1988) and Ahmed, (1994). 


\section{REFERENCES}

Ahmed, Z.A. (1994). Preliminary survey of soil algal flora in uppe Egypt. Egyptian Journal of Botany. 34 (1): 17-36.

Allea, S.E. (1989). Chemical analysis of ecological materials. Second Edition, Blackweel Scientific Publications, Oxford. PP 36.

Allen, S.E.; Grinshaw, H.M.; Parkinson, J.A. and Quarmby, C. (1974). Chemical analysis of ecological materials. In: Allen, S.E. (ed.). Blackwell Scientific Publications, Oxford. PP. 565.

Ashley, J.; Rushforth, S.R. and Johansen, J.R. (1985). Soil-Algae of Cryptogamic Crusts from the Unitah Basin, Utah, U.S.A. Great Basin Naturalist. 45 (3): 312-318.

Bock, W. (1963). Diatomeen extrem trockener standorte. New Hedw. 6:199-254.

Bold, H.C. (1970). Some aspects of the Taxonomy of Soil algae. Ann New York Acad Sci. 175:601-616.

Bold, H. C. and Wynne, M.J. (1985). Introduction to the algae: Structure and Reproduction. $2^{\text {nd }}$ ed. Prentice-Hall, Englewood Cliffs, NJ. PP 706.

Brock, T.D. (1973). Primary colonization of surtsey with special reference to the blue-green Algae. Oikos. 24:239-243.

Cano, M.S.; Mule, M.C.Z.; de Caire, G.Z.; de Palama, R.M. and Colombo, K. (1997). Aggregation of Soil Particles by Nostoc muscorum Ag. (Cyanobacteria). Internationa J. of Experimental Botany. 60 (2): 33-38.

Chapman, V.J. (1962). The algae St. martin's Press. Mocmillan, New York U.S.A. PP. 105.

Desikachary, T.V. (1959). Cyanophyta. Indian Council of Agricultural Research, New Delhi.

Deutsche Einheitsver Fahren Zur Wasser-Abwasser-und Schlammunter. Suchung Verlag Chemie, Weinhum (1960).

El-Ayouty, A.Y. and Ayyad, M.A. (1972). Studies on Blue-Green Algae of the Nile Delta. 1. Description of Some species in a Wheat Field. Egypt. J Bot. 15:283.

El-Kheir, W.S.A. and Mekkey, L.E. (1987). Notes on soil Algae in Different Regions in Egypt. Phytologia. 6 (7):429-433.

Fogg, G.F.; Stewart, W.D.P.; Fay, P. and Walbsy, A.E. (1973). The blue-green algae. Academic Press, New York.

Fritsch, F.E. (1936). The Role of the Terrestrial alga in nature. Essays in Geobotany in Honor of W.A. Setchell. PP 195-217.

Hoffmann, L. (1989). Algae of Terresterial Habitats. The Bot Rev. 55 (2): 77 105.

Hong, Y., Li, Y.Y. and Li, S.H. (1992). Preliminary study on the blue-green algal community of arid soil in Qaidam basin. Acta Botanica Sinica. 34 (3): 162-168.

John, R.P. (1942). Ecological and Taxonomic study of the algae of British soil I. The distribution of the surface growing algae. Ann. Bot. 6:323-349.

Egyptian J. of Phycol. Vol. 4(1), $2003 \quad$ - 52 - 
Kobbia, I.A. and El-Batanouny, K.H. (1975). Studies on the algal flora of egyptian soils. I-different sites along a triklet of a lake in the salines of wadi-El-Natroun. Publ. Cairo Univ. Herb. 6:61.

Kobbia, I.A. and Shabana, E.F. (1988). Studies on the soil algal flora of egyptian Bahariya Oasis. Egyptian. J. Bot. 31 (1-3): 23-44.

Lukesova, A. and Hoffmann, L. (1996). Soil algae from acid rain impacted forest areas of the krusne hory mountains 2. Effect of $\mathrm{pH}$ on Growth. Archiv Fuer Hydrobiologie supplementband. 109 (10): 39-51.

Mandal, R.; Begum, Z.N.T.; Khan, Z.U.M. and Hossain, M.Z. (1993a). N2fixing blue-green algae in rice fields and their relationship with soil fertility. Bangladesh. J Bot. 22 (1): 73-79.

Mandal, B.; Das, N. and Gosh, R. (1993b). Relative efficiency of Azolla and Other Organic manures in Summer Rice Oryza Sativa. Indian J of Agric Sci 63 (4):195-199.

Mandal, B.; Das, N. and Dhosh, R. (1993c). Relative efficiency of azolla and other organic manures in winter rice oryza sativa. Indian J of Agric Sci. $\mathbf{6 3}$ (4): 340-344.

Metting, B. (1981). The systematic and ecology of soil algae. The Bot. Rev. 47 (2): 195-312.

Ohtani, S.; Akiyama, M. and Kanda, H. (1991). Analysis of Antarctic soil algae by the direct observation using contact slid method. Antarctic record. 35 (3): 285-295.

Petersen, J.B. (1935). Studies on the biology and taxonomy of soil algae. Dansk. Bot Arkiv. 8:1-180.

Piper, C.S. (1974). Soil and plant analysis. The University of Adelaide, Adelaide. PP. 368.

Prescott, G.W. (1962). Algae of Western Great Lakes Area. W.M.C. Brown Co. Inc. Dubuque. Iowa. U.S.A.

Prescott, G.W. (1970). How to known the fresh water algae. William C. Brown Company, Dubuque, Iowa.

Rodrignez, H.; Rivas, J.; Guerrero, M.G. and Losada M. (1990). Calcium Ion Requirement for aerobic nitrogen fixation by heteroscystous blue-green algae. Plant Physiology (Bethesda). 92 (4): 886-890.

Rogers, P.A. and Reynaud, P.A. (1979). Enumeration of the algae in submerged soi: Law of the distribution of organisms and the density of Sampling. Rev. Ecol. Biol. Soil. 15:219-234.

Salama, A.M. and Kobbia, I.A. (1982). Studies on the algal flora of Egyptian Soils II. DIfferent sites of a sector in the lybian desert. Egyptian. J Bot. 25 No. 1-3.

Salama, A.M.; EL-Batanouny, K. and ALi, M.I. (1973). Studies on the fungal flora of Egyptian soils. I. West Mediterranean Coast. and Lybian Desert. U.A.R.J. Bot. 14:99. 
Staub, R. (1961). Ernahrungsphysioolohisch-autokologisch Untersuchungen an der planktischen blaulage oscillatoria rubescens DC. Schweiz. Z Hydrol. 22:82-198.

Watanabe, M. and Niiyama, Y. (1990). Fresh Water Algae from lake akan (5). Bull. Natn. Sci. Nus. Tokyo. Ser. B. 16 (1): 29-39.

Watanabe, M. (1992). Studies on planktonic blue-Freen Algae. 4. Some Anabaena Species with straight trichomes in Japan. Bull. Natu. Sci. Nus. Tokyo. Ser. B. 18 (4): 123-137.

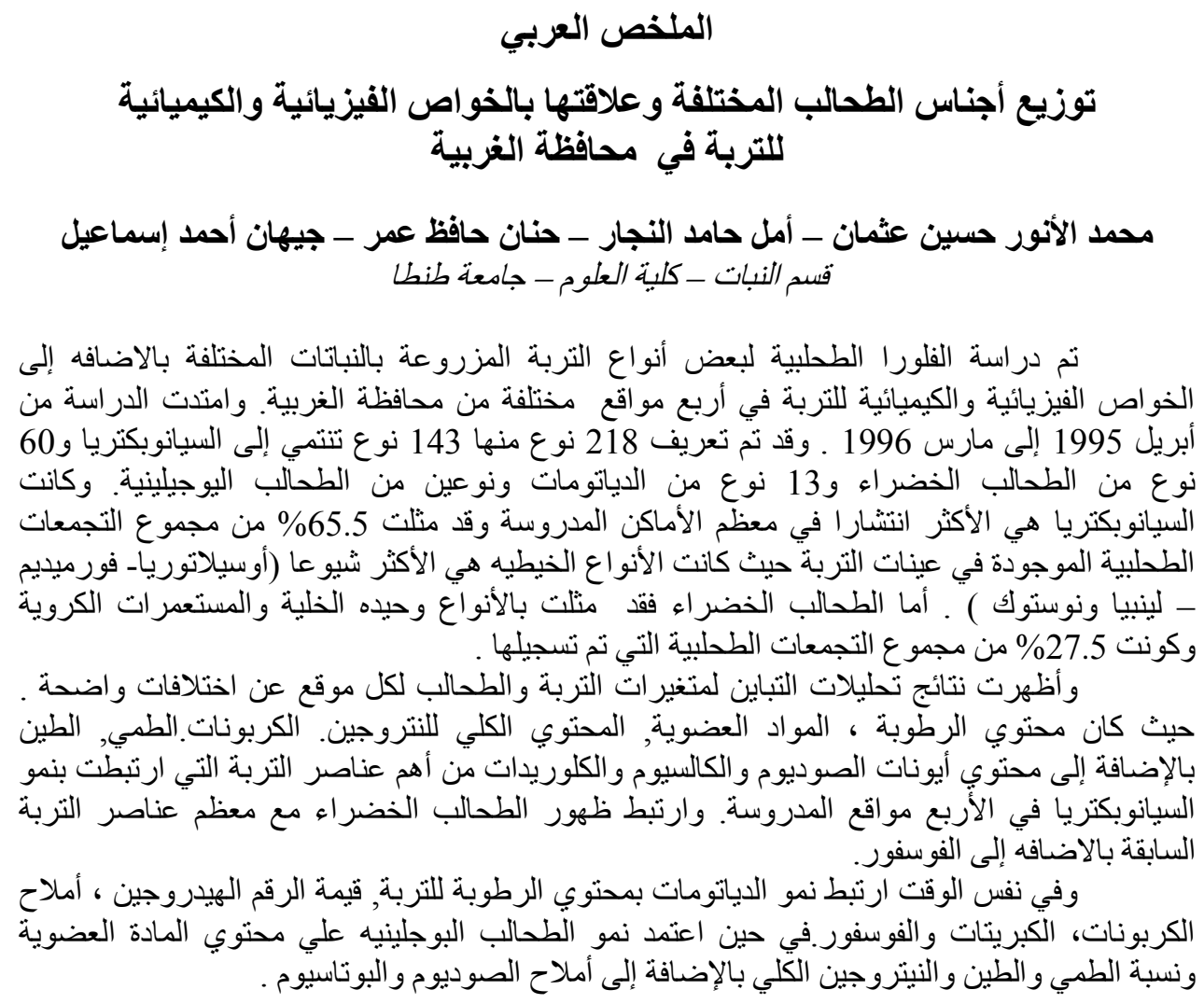

